\title{
Hyponatremia and cerebrovascular spasm in aneurysmal subarachnoid hemorrhage
}

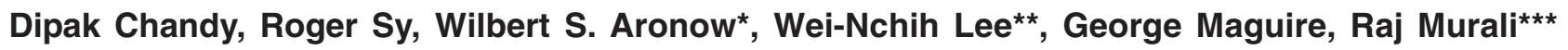 \\ Departments of Medicine Divisions of Pulmonary/Critical Care, ${ }^{*}$ Cardiology and ${ }^{* *}$ General Internal Medicine and ${ }^{* * *}$ Neurosurgery, \\ New York Medical College, Valhalla, NY, USA
}

Background: Hyponatremia develops in approximately a third of patients with aneurysmal subarachnoid hemorrhage $(\mathrm{SAH})$. Studies have been conflicting about the association between hyponatremia and cerebrovascular spasm (CVS). Aims: To investigate whether hyponatremia can signal the onset of CVS. Settings and Design: Retrospective chart review of all patients with SAH treated at a tertiary-care university hospital from January to May 2002. Materials and Methods: 106 patients were included in the study. Serum sodium levels were recorded from days 1 to 14 of hospitalization. Hyponatremia was defined as serum sodium level $<135 \mathrm{meq} / \mathrm{l}$ and a fall in sodium level of $>4$ meq/l from the admission sodium level. The presence of CVS was determined by transcranial doppler sonography. Patients were assigned to one of four groups based on the presence or absence of CVS and hyponatremia. Statistical Analysis: Student's t-test was used for comparison of means. A logistical regression model was constructed and odds ratios (OR) were calculated. Results: 41 patients developed hyponatremia and 44 developed CVS. Among the 41 with hyponatremia, 22 (54\%) had evidence of CVS, whereas among the 65 patients without hyponatremia, $22(34 \%)$ had evidence of CVS $(P=0.023)$. Among those with hyponatremia, the mean sodium drop was $7.9 \mathrm{meq} / \mathrm{L}$ in those with CVS compared to $7.0 \mathrm{meq} / \mathrm{L}$ in those without CVS $(P=0.068)$. More than half of those with hyponatremia and CVS (13/ 22) developed hyponatremia at least a day before CVS was diagnosed. Conclusion: In patients with SAH, hyponatremia is associated with a significantly greater risk of developing CVS and may precede CVS by at least one day.

Key words: Cerebral vasospasm, hyponatremia, subarachnoid hemorrhage

\section{Introduction}

Cerebrovascular spasm (CVS) causes symptomatic ischemia and infarction in approximately $20-50 \%$ of patients with aneurysmal subarachnoid hemorrhage (SAH) and is the leading cause of death and disability in these patients after aneurysm rupture. CVS usually occurs between days 3 and 14 after SAH and its onset is typically characterized by a decline in neurological status. Because changes in flow typically precede clinical changes, transcranial doppler sonography (TCD) can be used to detect the onset of CVS and is often performed daily to offer a window of opportunity for treating patients before clinical decline.

Hyponatremia after SAH is relatively common with prevalence rates of approximately $30-55 \% .^{[1]}$ The etiology of hyponatremia in SAH is unclear but is probably mediated by hypothalamic injury secondary to CVS. This injury leads either to increased secretion of antidiuretic hormone, which causes the syndrome of inappropriate antidiuretic hormone (SLADH) or to cerebral salt wasting (CSW), ${ }^{[2-5]}$ caused by enhanced release of atrial natriuretic peptide, ${ }^{[6,7]}$ brain natriuretic peptide ${ }^{[4,8,9]}$ dendroaspis natriuretic peptide, ${ }^{[10]}$ adrenomedullin ${ }^{[11]}$ or noradrenaline. ${ }^{[9]}$ Regardless of the etiology, hyponatremia, when present, potentially worsens the underlying neurological condition and severe hyponatremia probably increases the risk of mortality and morbidity. ${ }^{[12]}$

Hyponatremia has often been noted to precede the onset of clinically detectable CVS, although this relationship is not well established. In two retrospective studies, clinical CVS or delayed cerebral ischemia after SAH was found to be more frequent among patients who also developed hyponatremia. ${ }^{[12,13]}$ However, a more recent study by Qureshi, et al, ${ }^{[14]}$ concluded that the presence of hyponatremia was not associated with increased risk of symptomatic CVS. All these studies used a serum sodium level < $135 \mathrm{meq} / \mathrm{l}$ as the sole criterion to define hyponatremia. To our knowledge, no study has used the amount of change of the serum sodium level as an additional criterion for hyponatremia. 
Furthermore, it is not clear if hyponatremia, when it occurs, predates the diagnosis of CVS and if its severity is related to the development of CVS.

In this study, we sought to determine if a correlation exists between our more rigid definition of hyponatremia and the occurrence of clinical CVS. If a correlation between hyponatremia and clinical CVS is present, we hoped then to determine if the hyponatremia predates the CVS and if the development of CVS is related to the magnitude of change in the serum sodium levels.

\section{Materials and Methods}

\section{Study population and design}

This retrospective study was approved by the New York Medical College Institutional Review Board. The medical records of all patients, regardless of Glasgow Coma Score, admitted to Westchester Medical Center with spontaneous SAH between January and May 2002 were reviewed. These records were made available to us by our medical records department and the data was retrieved exclusively by one of the authors. All patients in whom an aneurysm was detected and a decision was made to intervene, underwent clipping as coiling was not being performed at our institution at the time of the study. Blood pressure and fluid management was according to an ICU protocol and therefore was relatively uniform across patients.

Patients were excluded from the study for any of the following reasons: 1 ) age $<18$ years; 2) SAH secondary to trauma or evidence of mycotic aneurysms; 3) death within 10 days of hospitalization; 4) concomitant severe medical illnesses like decompensated heart failure, advanced liver disease, renal failure and untreated hypothyroid or adrenal insufficiency.

\section{Data collection}

TCD was performed on patients almost on a daily basis. Patients were considered to have CVS if TCD readings $(>120 \mathrm{~cm} / \mathrm{s}$ in the anterior and middle cerebral artery territory) were consistent with that diagnosis.

Because almost all cases of CVS occur in the first 2 weeks after $\mathrm{SAH},{ }^{[15]}$ the serum sodium levels of each patient in the study were reviewed from day 1 to day 14 of hospitalization. If the serum sodium level was measured more than once on a day, then the lowest level for that day was used for purposes of analysis. For this study, hyponatremia was defined as both an absolute value of $<135$ meq/l occurring at any point in time during the first 14 days of hospitalization and a decrease from the admission serum sodium level (day 1 ) of $>4$ meq/l. The latter criterion was added to the more traditional definition of hyponatremia to avoid defining patients as being hyponatremic when their serum sodium levels fell below the lower limit of normal but the change was very small. We believe that such changes are not clinically significant. Thus, a patient with an admission serum sodium level of 136 meq/l that dropped to a low of $134 \mathrm{meq} / \mathrm{l}$ on day 5 was considered not to have hyponatremia.

\section{Data analysis}

Patients were divided into 4 groups according to the presence or absence of hyponatremia and the presence or absence of CVS. Among the patients with hyponatremia, the mean maximum decrease in the serum sodium level of patients with CVS was compared with that of patients without CVS. Student's t-test was used for comparison of means. A logistical regression model was constructed with CVS as the outcome and hyponatremia as a bivariate independent predictor variable. An age and sex-adjusted odds ratio $(\mathrm{OR})$ was calculated. Significance was set as $P<0.05$.

\section{Results}

The charts of 177 patients with SAH were reviewed and 106 patients were identified for inclusion in the study. Of the 71 patients excluded, 27 had non-aneurysmal bleeding, 21 had fewer than 10 days of hospital survival or a "do not resuscitate" status, 10 were transferred to other institutions in the first 2 weeks, 9 had coexisting severe medical conditions and 4 were under 18 years of age. Of the 106 patients included, $63(59.4 \%)$ were female and $43(40.6 \%)$ were male. The mean age \pm SD was $53 \pm 15$ years (range, 18 to 88 years). The mean admission serum sodium was $138.1 \pm 3.3 \mathrm{meq} / \mathrm{dl}$ (range, 126 to $149 \mathrm{meq} / \mathrm{dl}$ ).

Forty-one of the 106 patients (39\%) developed hyponatremia and 44 of the 106 (42\%) developed CVS. Among the 41 patients who developed hyponatremia, 22 patients had evidence of CVS (54\%), whereas among the 65 patients who did not develop hyponatremia, $22(34 \%)$ had evidence of CVS [Table 1]. This difference was statistically significant $(P=0.023 ; \mathrm{OR}=2.67$, CI $=1.2-6.2$ ). The mean maximum decrease in serum sodium level among those with hyponatremia and CVS was $7.9 \mathrm{meq} / \mathrm{L}$ and among those with hyponatremia and without CVS was $7.0 \mathrm{meq}$ $\mathrm{L}$; this difference was not statistically significant $(P=0.068)$. Among the 22 patients with CVS, hyponatremia occurred at least one day before clinical CVS was diagnosed in 13 (59\%); only 4 cases occurred after the detection of CVS.

\section{Discussion}

In this study population, hyponatremia occurred more frequently among patients with SAH who developed clinical CVS during the first 14 days of hospitalization. Those patients with SAH who developed hyponatremia were 2.6 times more likely to develop clinical CVS. In addition, hyponatremia preceded the detection of CVS by TCD, thereby potentially providing an earlier marker of CVS. However, the magnitude of the mean maximum decrease in serum sodium level was not a significant predictor for

Table 1: Number of cases with and without hyponatremia and CVS

\begin{tabular}{lcccc}
\hline & CVS & No CVS & OR $^{*}$ & $\boldsymbol{P}_{\text {value }}$ \\
Hyponatremia & 22 & 19 & 2.67 & 0.023 \\
No hyponatremia & 22 & 43 & & \\
\hline
\end{tabular}

CVS $=$ Cerebrovascular spasm, *Odds ratio adjusted for age and sex. 
the development of CVS.

A study previously identified four independent predictors of symptomatic CVS: thickness of the subarachnoid clot on CT scan, middle cerebral artery mean flow velocity $\geq 110 \mathrm{~cm} / \mathrm{sec}$ on or before day 5 post-hemorrhage, Glasgow Coma Score $<14$ and rupture of anterior cerebral artery or internal carotid artery aneurysm. ${ }^{[16]}$ Our study has potentially identified an additional parameter, the presence of hyponatremia, to determine the risk of developing CVS. Recognition of this association could lead to earlier treatment of CVS, which in turn might lead to a decrease in the mortality and morbidity rates.

Despite the more stringent definition of hyponatremia used in our study, our observations were comparable to the observations made by Widjicks, et al. ${ }^{[12]}$ In their study $60 \%$ of patients with hyponatremia developed CVS whereas it was $12 \%$ in patients with normal serum sodium levels. However in the study by Hasan, et $a l,,^{[13]}$ only $24 \%$ of patients with hyponatremia developed CVS. In a more recent study, Qureshi, et $a l,{ }^{[14]}$ did not find any correlation between hyponatremia and the risk of CVS. However, in this study serum sodium levels were measured daily for the first 2 weeks as was done in our study.

The limitations of our study include the fact that it was a retrospective study at a single center, TCDs were not performed daily for some patients and that a number of patients were excluded for a variety of reasons. Because most of our patients were initially admitted to community hospitals and were transferred to our institution several hours to 2 days after the onset of symptoms, it is possible that our day one serum sodium levels may not be truly representative of the level at the time of the SAH. While our ICU management protocol allows for the administration of only isotonic solutions, some patients may have been given hypotonic solutions before being transferred to our institution. Possibly the biggest limitation of the study is that the precise sodium intake by each patient could not be measured. Despite the fact that normal saline $(0.9 \%)$ was the only type of intravenous fluid used, patients were usually receiving different types and amounts of nutrition and water enterally, which may have had an independent impact on the sodium levels.

\section{Conclusions}

This study showed a good correlation between the presence of hyponatremia and the risk of developing CVS in patients with
SAH. However, the severity of the hyponatremia did not appear to predict the development of CVS. Most cases of hyponatremia occurred before the detection of CVS, thereby suggesting that hyponatremia could be used as an additional early marker of CVS in conjunction with the four independent predictors of CVS already identified.

\section{References}

1. Sherlock M, O'Sullivan E, Agha A, Behan LA, Rawluk D, Brennan P, et al. The incidence and pathophysiology of hyponatraemia after subarachnoid haemorrhage. Clin Endocrinol 2006 64:250-4.

2. Isotani E, Suzuki R, Tomita K, Hokari M, Monma S, Marumo F, et al. Alterations in plasma concentrations of natriuretic peptides and antidiuretic hormone after subarachnoid hemorrhage. Stroke 1994 25:2198-203.

3. Nelson PB, Seif SM, Maroon JC, Robinson AG. Hyponatremia in intracranial disease: Perhaps not the syndrome of inappropriate secretion of antidiuretic hormone (SLADH). J Neurosurg 1981;55:938-41.

4. Revilla-Pacheco FR, Herrada-Pineda T, Loyo-Varela M, Modiano-Esquenazi M. Cerebral salt wasting syndrome in patients with aneurysmal subarachnoid hemorrhage. Neurol Res 2005 27:418-22.

5. Wijdicks EF, Vermeulen M, ten Haaf JA, Hijdra A, Bakker WH, van Gijn J. Volume depletion and natriuresis in patients with ruptured intracranial aneurysm. Ann Neurol $1985 ; 18: 211-6$

6. Sviri GE, Feinsod M, Soustiel JF. Brain natriuretic peptide and cerebral vasospasm in subarachnoid hemorrhage. Clinical and TCD correlations. Stroke 2000;31:118-22.

7. Wijdicks EF, Ropper AH, Hunnicutt EJ, Richardson GS, Nathanson JA. Atrial natriuretic factor and salt wasting after aneurysmal subarachnoid hemorrhage. Stroke 1991;22:1519-24.

8. Berendes E, Walter M, Cullen P, Prien T, Van Aken H, Horsthemke .J, et al. Secretion of brain natriuretic peptide in patients with aneurysmal subarachnoid haemorrhage. Lancet 1997;349:245-9.

9. Tomida M, Muraki M, Uemura K, Yamasaki K. Plasma concentrations of brain natriuretic peptide in patients with subarachnoid hemorrhage. Stroke 1998;29:1584-7

10. Khurana VG, Wijdicks EF, Heublein DM, McClelland RL, Mever FB, Piepgras DG, et al. A pilot study of dendroaspis natriuretic peptide in aneurysmal subarachnoid hemorrhage. Neurosurgery 2004;55:69-75.

11. Wijdicks EF, Heublein DM, Burnett JC Jr: Increase and uncoupling of adrenomedullin from the natriuretic peptide system in aneurysmal subarachnoid hemorrhage. J Neurosurg 2001;94:252-6.

12. Wịdicks EF, Vermeulen M, Hịdra A, van Gijn J. Hyponatremia and cerebral infarction in patients with ruptured intracranial aneurysms: is fluid restriction harmful? Ann Neurol 1985;17:137-40.

13. Hasan D, Wijdicks EF, Vermeulen M. Hyponatremia is associated with cerebral ischemia in patients with aneurysmal SAH. Ann Neurol 1990;27:106-8.

14. Qureshi AI, Suri MF, Sung GY, Straw RN, Yahia AM, Saad M, et al. Prognostie significance of hypernatremia and hyponatremia among patients with aneurysmal subarachnoid hemorrhage. Neurosurgery 2002;50:749-56.

15. Harrigan MR. Cerebral salt wasting syndrome: A review. Neurosurgery 1996;38:152-60.

16. Qureshi AI, Sung GY, Razumovsky AY, Lane K, Straw RN, Ulatowski JA. Early identification of patients at risk for symptomatic vasospasm after aneurysmal SAH. Crit Care Med 2000;28:984-90.

Accepted on 31-05-2006 